\title{
Link-Level Resource Allocation for Flexible-Grid Nonlinear Fiber-Optic Communication Systems
}

\author{
Li Yan, Erik Agrell, Senior Member, IEEE, Henk Wymeersch, Member, IEEE, Pontus Johannisson, \\ Rocco Di Taranto, Member, IEEE, and Maïté Brandt-Pearce, Senior Member, IEEE
}

\begin{abstract}
Resource allocation for transport optical networks has traditionally used a transmission reach constraint to estimate physical impairments. For flexible-grid networks, nonlinear impairments are not sufficiently well characterized by the transmission reach since various modulations and bandwidths can coexist on the same link. We propose a resource allocation algorithm for a single flexible-grid fiber link based on a nonlinear signal distortion model, as a first step towards whole-network design. An optimization problem is formulated to provide a close-to-optimal resource allocation that guarantees the quality of transmission for every channel. Compared with a simpler algorithm based on transmission reach, our proposed algorithm shows the potential of bandwidth savings. It is demonstrated to be insensitive to channel ordering.
\end{abstract}

Index Terms-Resource allocation, nonlinear channel.

\section{INTRODUCTION}

$\mathbf{F}$ LEXIBLE grid optical networks can significantly increase the utilization of optical networks by allocating resources adaptively according to the traffic demands [1]. This requires the network planning to accurately estimate the physical layer impairments (PLI), including amplified spontaneous noise (ASE) and nonlinear interference (NLI).

Previous approaches to estimate PLI and guarantee the quality of transmission (QoT) for networks have relied on the transmission reach [1], [2], which is a suitable method for network planning in a dynamic environment. However, in flexible-grid networks, signals with various modulations and bandwidths can share the same link, and the PLIs are notably more state-dependent than in fixed-grid networks. The various approaches to bounding the transmission reach presented in recent literature do not address this problem. In [1], [3], each channel's PLI is calculated individually assuming a worstcase scenario, leading to overestimated NLI. This has been addressed in [2], where a transmission reach model including NLI within a certain spectral range is used in a mixed line rate wavelength-division multiplexing (WDM) network. However, this method is sub-optimal because the simplified PLI model does not have control of the power of each channel, and the transmission reach estimation is still conservative. Recently, a more sophisticated analytical PLI model, called Gaussian noise (GN) model [4], [5] has been proposed which combines reasonable accuracy and low computational complexity. Based on the GN model, a more accurate transmission reach estimation for Nyquist WDM superchannels has been calculated to achieve savings of resources in flexible optical networks [6].

Research supported by the Swedish Research Council under Grants No. 2011-6864 and 2012-5280. L. Yan, E. Agrell, H. Wymeersch, R. Di Taranto, and P. Johannisson are with Chalmers University of Technology, SE-41296 Gothenburg, Sweden (lyaa@ chalmers.se). M. Brandt-Pearce is with University of Virginia, Charlottesville, 22904 Virginia, USA.
This approach uses a fixed ratio between channel spacing and bandwidth, thus is not flexible enough to fully exploit the benefits introduced by fine granularity wavelength-selective switches. The GN model has also been used in fixed-grid network optimization [7] and congestion-aware routing [8].

The algorithms above can be divided into two categories: (i) off-line optimization that allocates resources jointly for static traffic demands [1], [2], [6], [7] and (ii) real-time planning that deals with dynamic traffic demands in a sequential manner [3], [8]. In this paper, we only consider the off-line planning problem, thus will not compare with dynamic algorithms.

In this paper we utilize a combined PLI model [4], [9] to optimize the allocation of resources, such as modulation formats, power spectral densities (PSDs), and carrier frequencies, to traffic requests for a single flexible-grid link. Understanding this link-level optimal allocation will enable more robust and efficient whole-network design. A close-tooptimal solution is provided by the optimization formulation, using an accurate prediction of PLI guaranteed by the physical model. Because only a single link is studied in this paper, we compare our method with an extended version of the reachbased method [6] instead of other network-level algorithms.

\section{Physical Layer Model and Problem Statement}

\section{A. Physical Layer Model}

The GN model [4], [5] allows approximate calculation of the PLI and the signal-to-noise ratio (SNR) for all connections in a network [9]. The model assumes (i) a coherent polarizationmultiplexed system without inline compensation for chromatic dispersion; (ii) for each channel, the transmitted signal PSD is equal in both polarizations; (iii) each channel spectrum is rectangular and does not overlap with neighboring channels; (iv) the NLIs generated in different fiber spans sum incoherently over the whole link; (v) the loss of each fiber span is compensated by an EDFA at the end of the span; (vi) all spans are long enough to have the same effective length; (vii) multichannel interference (MCI) [4], [9] is neglected. The NLI PSD per polarization for channel $i$ can then be expressed as [4], [9]

$$
\begin{aligned}
G_{i}^{\mathrm{NLI}}= & \mu G_{i}\left(G_{i}^{2} \operatorname{arcsinh}\left(\rho \Delta f_{i}^{2}\right)\right. \\
& \left.+\sum_{\substack{j=1 \\
j \neq i}}^{M_{\mathrm{c}}} G_{j}^{2} \ln \left|\frac{\left|f_{i}-f_{j}\right|+\Delta f_{j} / 2}{\left|f_{i}-f_{j}\right|-\Delta f_{j} / 2}\right|\right),
\end{aligned}
$$

where $\mu=3 \gamma^{2} /\left(2 \pi \alpha\left|\beta_{2}\right|\right), \rho=\pi^{2}\left|\beta_{2}\right| / 2 \alpha, M_{\mathrm{c}}$ is the number of channels or calls, $\gamma$ is the nonlinear parameter, $\alpha$ is the fiber power attenuation, $\beta_{2}$ is the group velocity dispersion (GVD) parameter, and $\Delta f_{k}, f_{k}$, and $G_{k}$ are the 
$k^{\text {th }}$ channel's bandwidth, center frequency, and signal PSD per polarization, respectively. The first term in (1) represents selfchannel interference (SCI) and the summation term is crosschannel interference (XCI). The logarithm function in the SCI term in [9, Eq. (16)] is replaced with the inverse hyperbolic sine function [4, Eq. (43)] for higher accuracy when $\Delta f_{i}$ is small, while they are the same for large $\Delta f_{i}$.

In addition to the NLI, the channel suffers from white Gaussian noise added by each EDFA with PSD $G^{\mathrm{ASE}}=$ $\left(e^{\alpha L}-1\right) h \nu n_{\mathrm{sp}}$, where $L$ is the fiber length per span, $n_{\mathrm{sp}}$ is the spontaneous emission factor, $\nu$ is the light frequency, and $h$ is Planck's constant. Combining the linear and nonlinear noise, the SNR for channel $i$ can be approximated as

$$
\mathrm{SNR}_{i}=\frac{G_{i}}{\left(G^{\mathrm{ASE}}+G_{i}^{\mathrm{NLI}}\right) N_{\mathrm{s}}},
$$

where $N_{\mathrm{s}}$ is the number of spans in the fiber link. In this paper, we set $\alpha=0.22 \mathrm{~dB} / \mathrm{km}, L=100 \mathrm{~km}, n_{\mathrm{sp}}=1.8$, $\gamma=1.32 \times 10^{-3}(\mathrm{Wm})^{-1}, \beta_{2}=-21.7 \mathrm{ps}^{2} / \mathrm{km}$, and $\nu=$ $193.55 \mathrm{THz}$.

\section{B. Problem Statement}

Our objective is to allocate a minimum of resources on a single link for a set of traffic demands with acceptable QoT. We assume that all the channels have the same PSD $\left(G_{i}=G\right)$, which makes the solution sub-optimal but reduces the computation complexity significantly. We denote the $i^{\text {th }}$ channel's traffic demand by $R_{i}$ (in Gbps), which includes forward error correction and operational overhead. The spectral efficiencies of available modulation formats are $c_{i} \in \mathcal{C}$, where $\mathcal{C}$ is a finite set $(\mathcal{C}=\{2,4,8\} \mathrm{bit} / \mathrm{s} / \mathrm{Hz}$ in this paper). Thus the bandwidth of channel $i$ using modulation format $c_{i}$ is $\Delta f_{i}=R_{i} / c_{i}$, assuming Nyquist pulse shaping. To obtain a satisfactory QoT, $\mathrm{SNR}_{i} \geq \mathrm{SNR}_{\mathrm{th}}\left(c_{i}\right)$ should be satisfied for all $i=1, \ldots, M_{c}$, where $\operatorname{SNR}_{\mathrm{th}}\left(c_{i}\right)$ is the $\mathrm{SNR}$ required to obtain a certain bit error rate (chosen to be $10^{-3}$ here) given the modulation format $c_{i}$. The center frequency of each channel is assumed flexible (no frequency grid). To extend the result in this paper to finite-grid networks, we can either round the carrier frequencies to the next multiple of the grid granularity, or solve the problem again with carrier frequencies as integer variables. Both methods will result in increased bandwidth utilization because more constraints are added. But the increase is marginal with modern switching technology that allows a spectrum resolution down to $3.125 \mathrm{GHz}$ [10].

\section{Solution StRategies}

We first present the optimization formulation, then give a transmission-reach method inspired by [6].

\section{A. Optimization Method}

The proposed resource allocation strategy can be formulated as an optimization problem. The objective is to minimize the spectrum usage, while guaranteeing the QoT. Mathematically, this is expressed as

$$
\begin{array}{cl}
\underset{\boldsymbol{c}, \boldsymbol{f}, G}{\operatorname{minimize}} & \frac{1}{2}\left(\frac{R_{1}}{c_{1}}+\frac{R_{M_{c}}}{c_{M_{c}}}\right)+f_{M_{c}}-f_{1} \\
\text { subject to } & \operatorname{SNR}_{i}(\boldsymbol{c}, \boldsymbol{f}, G) \geq \operatorname{SNR}_{\mathrm{th}}\left(c_{i}\right) \\
& K_{j}(\boldsymbol{c}, \boldsymbol{f}) \leq 0 \quad j=1, \ldots, M_{c}-1 \\
& c_{i} \in \mathcal{C} \quad i=1, \ldots, M_{c},
\end{array}
$$

where $\boldsymbol{c}=\left(c_{1}, \ldots, c_{M_{c}}\right)^{\mathrm{T}}$ is the vector of spectral efficiencies, $\boldsymbol{f}=\left(f_{1}, \ldots, f_{M_{c}}\right)^{\mathrm{T}}$ is the vector of center frequencies, and

$$
K_{j}(\boldsymbol{c}, \boldsymbol{f})=\frac{1}{2}\left(\frac{R_{j}}{c_{j}}+\frac{R_{j+1}}{c_{j+1}}\right)+f_{j}-f_{j+1},
$$

ensures that neighboring channels do not overlap.

Problem (3) is a mixed integer nonlinear program (MINLP). To solve it, we fix $G$ and relax the integer constraint in $c$. After some straightforward manipulation (3) can be expressed as

$$
\begin{array}{clr}
\underset{\boldsymbol{c}, \boldsymbol{f}}{\operatorname{minimize}} & \frac{1}{2}\left(\frac{R_{1}}{c_{1}}+\frac{R_{M_{c}}}{c_{M_{c}}}\right)+f_{M_{c}}-f_{1} \\
\text { subject to } & F_{i}(\boldsymbol{c}, \boldsymbol{f}) \leq 0 \\
& K_{j}(\boldsymbol{c}, \boldsymbol{f}) \leq 0 & \\
& c_{i} \in \operatorname{Conv}(\mathcal{C}) & i=1, \ldots, M_{c}-1 \\
&
\end{array}
$$

where

$$
\begin{aligned}
& F_{i}(\boldsymbol{c}, \boldsymbol{f})=G^{\mathrm{ASE}} S\left(c_{i}\right)+\mu G^{3} S\left(c_{i}\right) \operatorname{arcsinh}\left(\rho R_{i}^{2} / c_{i}^{2}\right) \\
& +\mu G^{3} S\left(c_{i}\right) \sum_{j \neq i} \ln \left(\frac{2\left|f_{i}-f_{j}\right| c_{j}+R_{j}}{2\left|f_{i}-f_{j}\right| c_{j}-R_{j}}\right)-G / N_{s},
\end{aligned}
$$

$S\left(c_{i}\right)$ is the required SNR threshold for $c_{i}$, and $\operatorname{Conv}(\mathcal{C})$ is the convex hull of $\mathcal{C}$, i.e., the smallest continuous interval on $\mathbb{R}$ that contains $\mathcal{C}$. Branch and bound is applied to (5) using the BONMIN [11] toolbox to search for the final mixed integer solution, where $c_{i} \in \mathcal{C}$ is enforced. The globally optimal solution is then found by repeating the above process for values of $G$ within a preset range.

\section{B. Transmission-Reach Method}

The transmission reach method in [6] is designed for homogeneous channels with the same bandwidth, modulation format, and guardband. However, heterogeneous channels are considered in this paper. So the method in [6] is modified as follows. We estimate the transmission reach based on a fixed number $\left(M_{\mathrm{c}}^{\mathrm{tr}}\right)$ of channels with the same modulation format $c$, bandwidth $\Delta f=R / c$, PSD $G$, and the ratio between channel spacing and bandwidth $\kappa=\left(f_{i+1}-f_{i}\right) / \Delta f \geq 1$. These parameters are plugged into the GN model (1) for NLI estimation. By varying $G$, the maximum number of spans $N_{\mathrm{c}}^{\text {tr }}$ that satisfies the QoT for all channels is defined as the transmission reach under the chosen parameters. The reach estimated based on $M_{\mathrm{c}}^{\mathrm{tr}}$, which can be expressed as

$$
\begin{aligned}
& N_{\mathrm{c}}^{\mathrm{tr}}\left(c, R, \kappa ; M_{\mathrm{c}}^{\mathrm{tr}}\right)= \\
& \max _{N_{\mathrm{s}}, G}\left\{N_{\mathrm{s}} \mid \operatorname{SNR}_{i}\left(G, N_{\mathrm{s}}\right) \geq \operatorname{SNR}_{\mathrm{th}}(c), i=1, \ldots, M_{\mathrm{c}}^{\mathrm{tr}}\right\},
\end{aligned}
$$

is precomputed and stored for all combinations of $\kappa, c$, and $R$. An example of $N_{\mathrm{c}}^{\mathrm{tr}}$ is illustrated in Fig. 1 with $M_{\mathrm{c}}^{\mathrm{tr}}=9$.

We adapt the transmission-reach method to heterogeneous traffic demands as follows. Given the length of the link $\left(N_{\mathrm{s}}\right)$, we determine the modulation format for each channel, and a common constant $\kappa$ for all the channels, such that the precomputed transmission reach of each channel is no less 


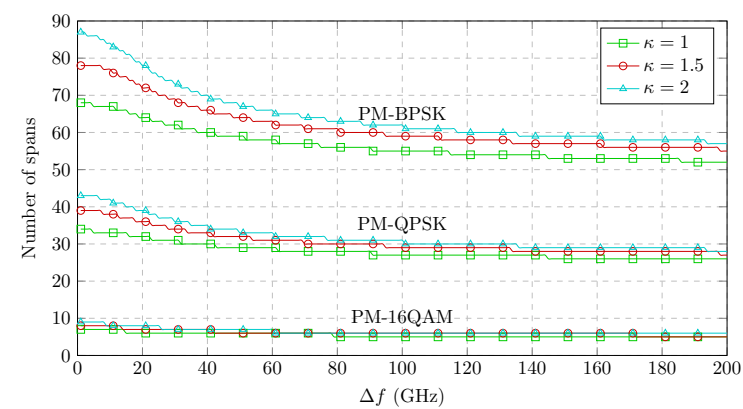

Fig. 1: The maximum reach as a function of the channel bandwidth $\Delta f=R / c$, modulation format $c$, and $\kappa$ estimated at $M_{\mathrm{c}}^{\mathrm{tr}}=9$ for homogeneous traffic. These values are precomputed and stored.

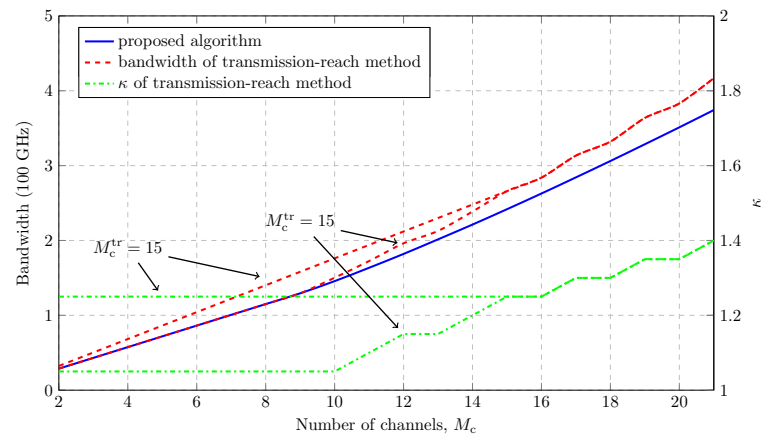

Fig. 2: The total bandwidth as a function of the number of channels for equal traffic demand of $115 \mathrm{Gbps}$ per channel. There are $N_{\mathrm{s}}=7$ spans, and the modulation formats used are all PM-16QAM.

than $N_{\mathrm{s}}$, and the total bandwidth is minimized. This is written as

$$
\begin{array}{cl}
\underset{c, \kappa \geq 1}{\operatorname{minimize}} & \frac{1}{2}\left(\frac{R_{1}}{c_{1}}+\frac{R_{M_{\mathrm{c}}}}{c_{M_{\mathrm{c}}}}\right)+\sum_{i=1}^{M_{\mathrm{c}}-1} \max \left\{g_{i}, g_{i+1}\right\} \\
\text { subject to } & N_{\mathrm{c}}^{\mathrm{tr}}\left(c_{i}, \kappa, R_{i} ; M_{\mathrm{c}}^{\mathrm{tr}}\right) \geq N_{\mathrm{s}} \\
& g_{i}=\kappa R_{i} / c_{i}, \quad i=1, \ldots, M_{\mathrm{c}},
\end{array}
$$

where $g_{i}$ is the channel spacing of channel $i$, and $c_{i} \in \mathcal{C}$. (8) is solved by exhaustive search in the precomputed transmission reach for $\kappa=\{1,1.05, \ldots, 4.95,5\}$. Observe in (8) that the precomputed reach uses $M_{\mathrm{c}}^{\text {tr }}$ channels, while the resources are allocated to $M_{\mathrm{c}}$ channels, and $M_{\mathrm{c}}^{\mathrm{tr}}$ is not necessarily equal to $M_{\mathrm{c}}$. The reason the objective minimizes $\max \left\{g_{i}, g_{i+1}\right\}$ is to address cases where two neighboring channels have different bandwidths. Note also that the solution to (8) may not satisfy the QoT requirements of the chosen modulation formats. To have a fair comparison with the optimization method, (8) is solved again excluding all the previously found solutions. This is repeated until a feasible solution is obtained. The PSD is set as the value that maximizes the minimum SNR of all $M_{\mathrm{c}}$ channels.

\section{NumericAl Results}

In this section, we show numerical results for the optimization method and compare with the transmission-reach method.

First, we compare the bandwidth allocated using different methods for channels with equal traffic demands. The results of the transmission-reach method are based on $M_{\mathrm{c}}^{\text {tr }}=9$ and $M_{\mathrm{c}}^{\text {tr }}=15$, respectively. In Fig. 2, all the cases turn out to use PM-16QAM for all the channels. $\kappa$ used by the transmission-reach method increases with $M_{\mathrm{c}}$, as larger

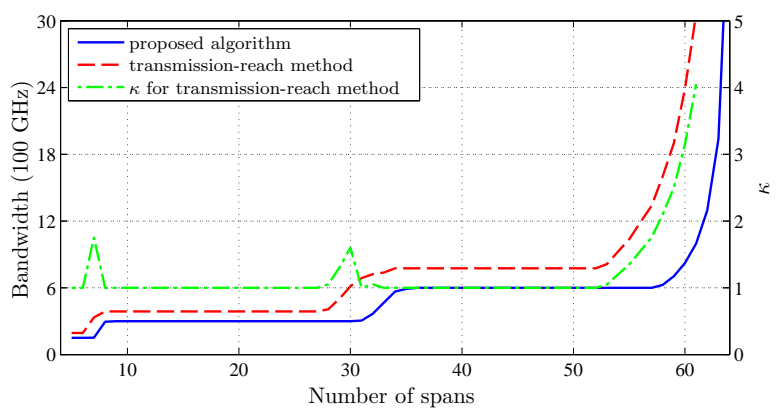

Fig. 3: The allocated bandwidth and $\kappa$ as functions of transmission distance. $M_{\mathrm{c}}^{\mathrm{tr}}=9$ is used in the transmission-reach benchmark.

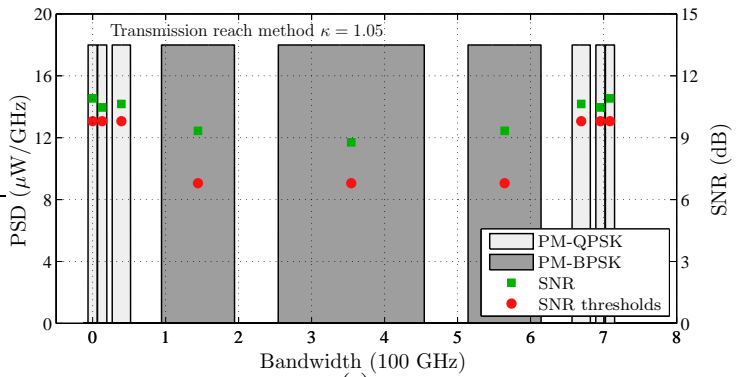

(a)

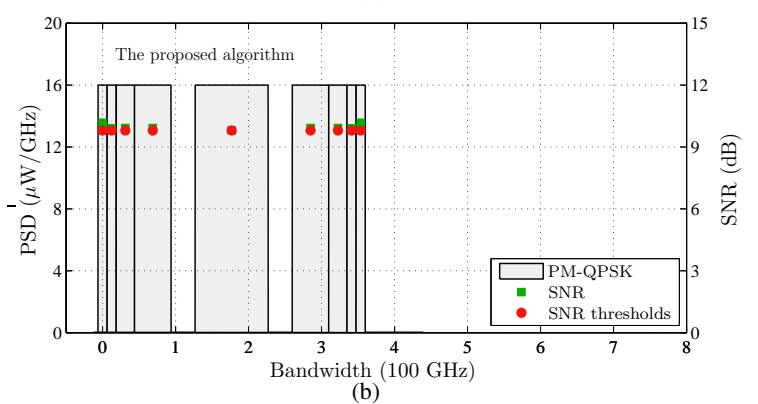

Fig. 4: The allocated spectra for $N_{\mathrm{s}}=32$ based on (a) the transmission-reach method and (b) the proposed algorithm.

$\kappa$ can tolerate more NLI. The bandwidth allocated by the optimization method is always less than the transmissionreach method. The bandwidth required by the transmissionreach method increases as $M_{\mathrm{c}}^{\text {tr }}$ increases, up to a limit. This shows how inaccurate the transmission-reach method is in NLI estimation as the actual number of channels varies, which results in inefficient bandwidth utilization in the end. Note that the reach-based method with $M_{\mathrm{c}}^{\mathrm{tr}}=15$ uses more bandwidth than the optimization method even at the $M_{\mathrm{c}}=15$ case. The reason is that the reach-based method requires a constant $\kappa$ for all the channels and thus reduces some degrees of freedom.

Second, 9 channels with heterogeneous traffic demands are considered according to the ordering 3 in Table I. Fig. 3 shows the allocated bandwidth and $\kappa$ as functions of the link length. Compared with the reach-based method, the optimization method leads to $22 \%$ spectrum utilization reduction. The switching from one plateau to another in the bandwidth curves corresponds to changes from one combination of modulation formats to another. At the switches, higher value of $\kappa$ is needed for the reach-based method to reduce the XCI.

To explore how the spectrum is actually allocated in Fig. 3, we focus on $N_{\mathrm{s}}=32$ and $N_{\mathrm{s}}=34$ and compare the optimization method with the transmission-reach method in Fig. 4 and 5, respectively. The reason for choosing $N_{\mathrm{s}}=32$ and $N_{\mathrm{s}}=34$ is that they are at the critical point of switching 


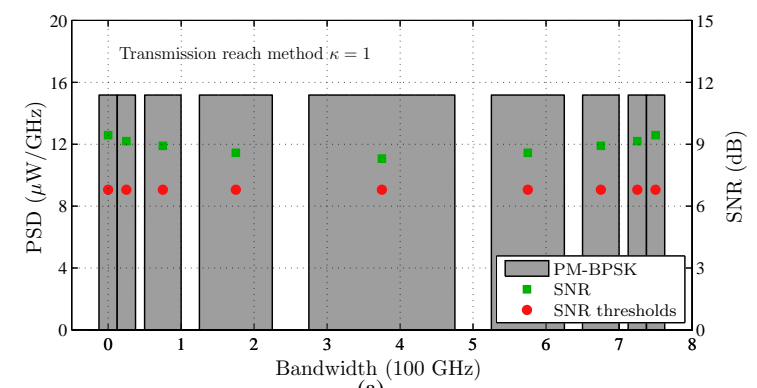

(a)

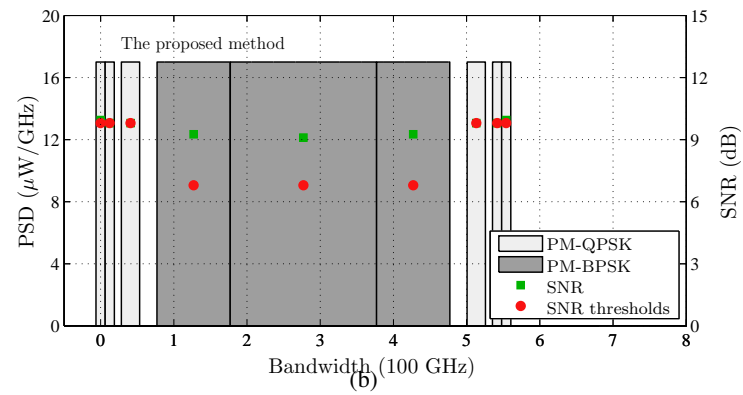

Fig. 5: The allocated spectra for $N_{\mathrm{s}}=34$ based on (a) the transmission-reach method and (b) the proposed algorithm.

TABLE I: Regular channel orderings

\begin{tabular}{|c|c|}
\hline Ordering & Traffic demands (Gbit/s) \\
\hline ordering 1 & $400,200,100,50,50,50,50,100,200$ \\
\hline ordering 2 & $50,50,50,50,100,100,200,200,400$ \\
\hline ordering 3 & $50,50,100,200,400,200,100,50,50$ \\
\hline
\end{tabular}

from one plateau to another in Fig. 3, and both examples have mixed modulation formats of PM-BPSK and PM-QPSK. Note that in the reach-based method (8), the modulation format of each channel is determined by both $\kappa$ and its data rate. As a result, in the heterogeneous case, mixed modulation formats are allowed in the reach-based method as well. Beyond $N_{\mathrm{s}}=34$, the outer 50 and $100 \mathrm{Gbps}$ channels still use PMQPSK, while the rest can only support PM-BPSK. This is because (i) channels with larger bandwidth have more SCI, and (ii) inner channels suffer from higher XCI than the outer ones. The colored markers illustrate each channel's actual SNR and SNR threshold. In both figures, the reach-based approach leaves unnecessarily large SNR margins while the optimization method is less conservative. By efficiently exploiting the SNR margins, the modulation formats and guardbands are allocated more flexibly and efficiently in the optimization method.

Note that orderings of the channels may result in different solutions to the optimization method as well. However, our study shows that the order of the calls has little impact on the required bandwidth unless the system is close to its operational limit. To illustrate this, we choose three different regular orderings from Table I, and 30 random orderings. Their allocated bandwidths as a function of transmission distance are given in Fig. 6. For most of the distances, all the orderings lead to similar bandwidth utilization. At the transitions (i.e., 6 to 9 spans, 30 to 36 spans, and 57 to 63 spans), ordering 1 and 2 are slightly better than the other orderings. Ordering 3 performs the worst among those tried. Part of the reason is that the central channel suffers from higher NLI, leading to a lower SNR, making it less able to use a higher-order modulation format than the outer channels (as shown in Fig. 5(b)).

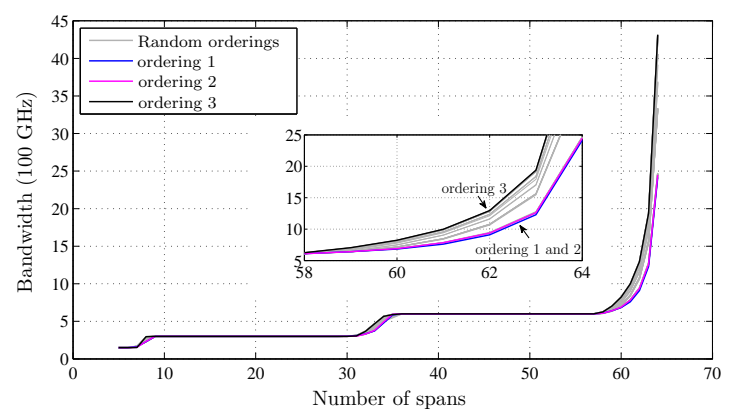

Fig. 6: The allocated bandwidth as a function of transmission distance for different orderings. The inset shows the allocated bandwidths from 58 spans to 64 spans.

Consequently, channels with smaller traffic demands should be arranged in the middle, so that their spectral efficiencies are sacrificed to save the total bandwidth.

\section{Conclusions}

In this paper, we have proposed a resource allocation algorithm that optimizes the bandwidth by allocating modulation formats, carrier frequencies, and PSDs jointly, based on the GN model. Compared with a heuristic transmission reach based method that considers different parameters separately, our scheme requires less bandwidth and achieves a longer reach. Moreover, the proposed method is based on rigorous optimization theory and is guaranteed to obtain the optimal solution. In contrast, case-sensitive tuning of parameters and formulations is needed for the reach-based method to find a satisfactory allocation.

\section{REFERENCES}

[1] K. Christodoulopoulos, I. Tomkos, and E. Varvarigos, "Elastic bandwidth allocation in flexible OFDM-based optical networks," IEEE $J$. Lightw. Technol., vol. 29, no. 9, pp. 1354-1366, 2011.

[2] K. Christodoulopoulos, K. Manousakis, and E. Varvarigos, "Reach adapting algorithms for mixed line rate WDM transport networks," IEEE J. Lightw. Technol., vol. 29, no. 21, pp. 3350-3363, 2011.

[3] J. Zhao, Q, Yao, X. Liu, L. Wei, and M. Maier, "Distance-adaptive routing and spectrum assignment in OFDM-based flexible transparent optical networks," Springer Photon. Netw. Commun., vol. 27, no. 3, pp. 119-127, 2014.

[4] P. Poggiolini, G. Bosco, A. Carena, V. Curri, Y. Jiang, and F. Forghieri, "The GN-model of fiber non-linear propagation and its applications," IEEE J. Lightw. Technol., vol. 32, no. 4, pp. 694-721, 2014.

[5] P. Johannisson and M. Karlsson, "Perturbation analysis of nonlinear propagation in a strongly dispersive optical communication system," IEEE J. Lightw. Technol., vol. 31, no. 8, pp. 1273-1282, 2013.

[6] E. Palkopoulou, G. Bosco, A. Carena, D. Klonidis, P. Poggiolini, and I. Tomkos, "Nyquist-WDM-based flexible optical networks: Exploring physical layer design parameters," IEEE J. Lightw. Technol., vol. 31, no. 14, pp. 2332-2339, 2013.

[7] D. J. Ives, P. Bayvel, and S. J. Savory, "Physical layer transmitter and routing optimization to maximize the traffic throughput of a nonlinear optical mesh network," in International Conference of Optical Network Design and Modeling, 2014.

[8] S. J. Savory, "Congestion Aware Routing in Nonlinear Elastic Optical Networks," IEEE Photon. Technol. Lett., vol. 26, no. 10, pp. 1057-1060, 2014.

[9] P. Johannisson and E. Agrell, "Modeling of nonlinear signal distortion in fiber-optic networks," IEEE J. Lightw. Technol., vol. 32, no. 23, pp. 3942-3950, 2014.

[10] O. Gerstel, M. Jinno, A. Lord, and S. Yoo, "Elastic optical networking: a new dawn for the optical layer?" IEEE Commun. Mag., vol. 50, no. 2, pp. s12-s20, February 2012.

[11] P. Bonami et al., "An algorithmic framework for convex mixed integer nonlinear programs," Elsevier Discret. Optim., vol. 5, no. 2, pp. 186 204, 2008. 